\title{
Evaluation of sexual history-based screening of anatomic sites for chlamydia trachomatis and neisseria gonorrhoeae infection in men having sex with men in routine practice
}

Remco PH Peters ${ }^{1,2^{*}}$, Stephan P Verweij ${ }^{3+}$, Noëmi Nijsten ${ }^{1}$, Sander Ouburg ${ }^{3}$, Johan Mutsaers ${ }^{4}$, Casper L Jansen ${ }^{4}$, A Petra van Leeuwen ${ }^{5}$ and Servaas A Morré ${ }^{3}$

\begin{abstract}
Background: Sexually transmitted infection (STI) screening programmes are implemented in many countries to decrease burden of STI and to improve sexual health. Screening for Chlamydia trachomatis and Neisseria gonorrhoeae has a prominent role in these protocols. Most of the screening programmes concerning men having sex with men (MSM) are based on opportunistic urethral testing. In The Netherlands, a history-based approach is used. The aim of this study is to evaluate the protocol of screening anatomic sites for C. trachomatis and $\mathrm{N}$. gonorrhoeae infection based on sexual history in MSM in routine practice in The Netherlands.

Methods: All MSM visiting the clinic for STI in The Hague are routinely asked about their sexual practice during consulting. As per protocol, tests for urogenital, oropharyngeal and anorectal infection are obtained based on reported site(s) of sexual contact. All consultations are entered into a database as part of the national STI monitoring system. Data of an 18 months period were retrieved from this database and analysed.

Results: A total of 1455 consultations in MSM were registered during the study period. The prevalence of $C$. trachomatis and $\mathrm{N}$. gonorrhoeae per anatomic site was: urethral infection $4.0 \%$ respectively and $2.8 \%$, oropharynx $1.5 \%$ and $4.2 \%$, and anorectum $8.2 \%$ and $6.0 \%$. The majority of chlamydia cases (72\%) involved a single anatomic site, which was especially manifest for anorectal infections (79\%), while $42 \%$ of gonorrhoea cases were single site. Twenty-six percent of MSM with anorectal chlamydia and $17 \%$ with anorectal gonorrhoea reported symptoms of proctitis; none of the oropharyngeal infections were symptomatic. Most cases of anorectal infection (83\%) and oropharyngeal infection (100\%) would have remained undiagnosed with a symptom-based protocol.
\end{abstract}

Conclusions: The current strategy of sexual-history based screening of multiple anatomic sites for chlamydia and gonorrhoea in MSM is a useful and valid guideline which is to be preferred over a symptom-based screening protocol.

Keywords: Chlamydia trachomatis, Neisseria gonorrhoeae, anorectal, oropharyngeal, screening

\section{Background}

Many countries have implemented national programmes for screening for sexually transmitted infections (STI). The aims of such programmes are to decrease the general burden of STI, to improve physical and sexual

\footnotetext{
* Correspondence: rph.peters@gmail.com

+ Contributed equally

'The Hague Municipal Health Services, STI clinic, The Hague, The Netherlands

Full list of author information is available at the end of the article
}

health, and to reduce transmission and acquisition of STI. Screening for chlamydia and gonorrhoea has a prominent place in these programmes and is done opportunistically, i.e. regardless of the presence of symptoms, because most of the cases are asymptomatic and there is a low diagnostic accuracy of selective screening criteria [1]. The screening of high-risk populations for urogenital infection has been shown to be feasible and is associated with clear reductions in the incidence of STI [2-6]. 
The core component of screening for Chlamydia trachomatis and Neisseria gonorrhoeae infection in MSM is testing for urogenital infection through urine sample or urethral swab. During the past decade, various studies from the United States of America (USA) and Australia have highlighted the importance of testing oropharyngeal and anorectal samples in addition to urogenital tests in MSM [7-14]. For example, a study in San Francisco showed that $53 \%$ of C. trachomatis and $64 \%$ of $N$. gonorrhoeae infections in MSM involved nonurethral sites and would be missed if screening was done only for urethral infection [7].

The present United Kingdom National Screening and Testing Guidelines, Norwegian HIV and STI screening protocols, Australian STI screening protocols, and guidelines from the Center for Disease Control and Prevention include specific recommendations for screening for anorectal and oropharyngeal infection in MSM based on reported site of sexual contact [4-6,15-17]. The Dutch Society for Dermatology and Venereology has similar guidelines for screening for chlamydia and gonorrhoea in MSM: a urethral or urine sample should be tested together with an oropharyngeal sample in case fellatio is reported and an anorectal sample if passive anorectal intercourse is reported. Anorectal infections in MSM have special attention due to the identification of the lymphogranuloma venereum strain L2b among MSM $[18,19]$. These tests should be obtained regardless of the presence of symptoms. In contrast to the UK and CDC guidelines, testing for oropharyngeal $C$. trachomatis infection is included in the Dutch guidelines as optional. Data on the prevalence of oropharyngeal chlamydia in MSM is limited, but in most studies the prevalence is $<2 \%$ [7,20-22].

At the STI clinic in The Hague the guidelines of the Dutch Society of Dermatology and Venereology are used for screening for chlamydia and gonorrhoea in MSM. These guidelines include anatomic site specific testing based on sexual history, including tests for oropharyngeal $C$. trachomatis infection. While anatomic site-specific STI testing in routine care has been evaluated previously in some countries, only small studies have been undertaken in Europe [7-14]. In addition, to our knowledge only two studies have been conducted in a European setting to assess the value of this approach, but these included a relatively small number of MSM (39 and 599, respectively) [21,22]. In the present study, we evaluate the protocol of screening multiple anatomic sites for chlamydia and gonorrhoea in a large cohort of MSM at an STI clinic in the Netherlands to assess the usefulness of this screening strategy in routine practice.

\section{Methods}

\section{Study population}

The STI Clinic in The Hague, The Netherlands offers free and anonymous STI screening to people living in the city of The Hague and the surrounding region. Approximately $15 \%$ of visitors to the clinic are MSM. During routine consultation, sexual history is obtained using a standard questionnaire. This questionnaire includes questions about STI history, sexual practice, number of sex partners, site(s) of sexual contact, and symptoms that may be associated with STI. After counselling, clients are routinely tested for chlamydia, gonorrhoea, syphilis, hepatitis B virus and HIV. The local medical ethical committee approved this study, based on the fact that in the Netherlands ethical approval is not required for a retrospective, de-identified study.

\section{Screening protocol}

The following protocol is used routinely for screening MSM for C. trachomatis and $N$. gonorrhoeae infection. First-void urine is collected from all asymptomatic visitors; a urethral swab is taken in case of penile discharge or dysuria. If fellatio is reported in sexual history, an oropharyngeal swab is obtained by wiping the swab twice over the lateral posterior sides of the pharyngeal wall followed by wiping once across the oropharyngeal wall. If receptive anal intercourse is reported, an anorectal sample is obtained by introducing a swab approximately $5 \mathrm{~cm}$ into the rectum under rotating movements. The swaps were clinician-collected without the use of a proctoscope.

\section{Data collection}

All consultations at the clinic are recorded in an anonymous electronic file. This record includes basic demographic data, the routine questionnaire, results of microbiological tests, diagnosis and treatment if applicable. After completion of the episode, all records are transferred anonymously into a national database (SOAP) for surveillance purposes. In this study, we retrieved and retrospectively analysed data from the SOAP database for the STI clinic in The Hague over an 18 month period (January 2007-July 2008).

\section{Clinical definitions}

We define urethral gonorrhoea or chlamydia as a positive test result for C. trachomatis or $N$. gonorrhoeae for a urethral swab, or urine sample by PCR. Urethral infection was considered symptomatic in case of dysuria or penile discharge. Pharyngeal chlamydia or gonorrhoea was considered symptomatic in case of a sore throat and rectal infection was defined symptomatic if anorectal discharge, blood or mucus in the stools, tenesmus or rectal pain was reported by the client.

\section{Laboratory tests}

Specific urethral, oropharyngeal and anorectal swabs were used for specimen collection $\left(\right.$ APTIMA $^{\circledR}$; Gen- 
Probe Incorporated, San Diego, CA). Samples from each anatomical site were collected in separate sample collection tubes. Testing of samples for $C$. trachomatis and $N$. gonorrhoeae was done using the APTIMA Combo $2^{\circledR}$ (AC2) assay (Gen-Probe Incorporated, San Diego, CA) according to the manufacturer's instructions. Positive reactions for $C$. trachomatis and $N$. gonorrhoeae with the AC2 assay were confirmed with the APTIMA CT (ACT) the APTIMA GC (ACG) assay respectively. Genotyping for specific detection of the lymphogranuloma venereum (LGV) strain was done on all anorectal samples with a positive result for C. trachomatis[23].

\section{Statistical analysis}

The SOAP database was evaluated for missing, incomplete and conflicting data and where possible cleaned by reviewing free text in the records. Data were analyzed using SPSS version 13.0 (SPSS Inc., Chicago, IL) and described as numbers (\%), proportion or median (range). Categorical data were compared between groups using Chi-square test and Fisher's Exact test when appropriate for categorical data and the Mann-Whitney test for continuous data. Risk factors were described as Odds ratio (OR) with 95\% confidence interval (CI). The distribution of prevalence of chlamydia and gonorrhoea per anatomic site was depicted stratified by 5 -year age groups.

\section{Results}

\section{Characteristics of study population}

During the study period a total of 1455 consultations by MSM were registered with complete data. This includes three transgender men who registered as women, but reported sexual contact with men and tests for male STI screening were obtained. The median age was 38 years old. The average sexual partners in the previous six months was four; 29 were commercial sex workers; 358 consultations had history of STI, and 128 were notified by a sexual partner. Clinical characteristics of the study population are summarised in table 1 .

\section{Prevalence of chlamydia and gonorrhoea per anatomic site}

Based on sexual history, tests for urethral infection were done in all except 4 consultations (99.7\%); 1283 (88\%) MSM reported fellatio and had oropharyngeal swabs taken while anorectal swabs were obtained from the MSM who reported passive anorectal intercourse ( $\mathrm{n}=$ $1141 ; 78 \%)$. In the majority of consultations $(n=1095)$ samples were obtained from all three anatomic sites $(75 \%)$, while $16 \%$ had two sites tested $(\mathrm{n}=230)$ and 130 (8.9\%) had only tests done for urethral infection.

The overall prevalence of chlamydia was $10 \%$ and prevalence of gonorrhoea was $7.7 \%$. The prevalence of $C$. trachomatis at the individual anatomic sites was: urethra $4.0 \%$, oropharynx $1.5 \%$ and anorectum $8.2 \%$, and for $N$. gonorrhoeae respectively $2.8 \%, 4.2 \%$ and $6.0 \%$ (Figure 1 ). Rectal C. trachomatis infection was diagnosed in 94 cases and the LGV strain was found in 5 (3.1\%); all of these presented with symptoms of proctitis.

The majority of C. trachomatis infections (72\%) involved a single anatomic site in those MSM with two or three anatomic sites tested. This was especially manifest for anorectal infection where $79 \%$ of cases were limited to the anorectum. Sixty-five percent of cases of urethral chlamydia and 53\% of those with oropharyngeal chlamydia were single site infections. In contrast, the majority of $N$. gonorrhoeae cases (59\%) involved multiple anatomic sites: only $26 \%$ of urethral, $43 \%$ of anorectal and $50 \%$ of oropharyngeal infections were limited to that specific anatomic site.

\section{Clinical presentation and risk factors}

Symptoms were reported in the minority of consultations: $16 \%$ urethral, $4.5 \%$ anal and $1.4 \%$ pharyngeal, while the majority of cases of chlamydia (56\%) and gonorrhoea $(43 \%)$ did report symptoms. Predictive values of specific STI symptoms for chlamydia and gonorrhoea were low: urethritis was reported by 228 men (16\%), but chlamydia was only diagnosed in 38 $(17 \%)$ and gonorrhoea in $34(15 \%)$. C. trachomatis infection was found in only 17 (26\%) and $N$. gonorrhoeae in $11(17 \%)$ of 65 individuals presenting with symptoms of proctitis. None of the cases with oropharyngeal chlamydia $(n=19)$ or gonorrhoea $(n=54)$ were symptomatic, but symptoms of pharyngitis were reported by $20 \mathrm{MSM}$ without chlamydia or gonorrhoea. As such, if a symptom-based protocol would have been used for screening of anorectum and oropharynx, 121/145 (83\%) of cases with anorectal infection and 68/68 (100\%) of those with oropharyngeal infection would have remained undiagnosed.

Median age of MSM with anorectal chlamydia or gonorrhoea was significantly higher than of those without anorectal infection (40 vs. 37 years; $\mathrm{p}=0.02)$; this association was not observed for urethral $(\mathrm{p}=0.6)$ or oropharyngeal $(\mathrm{p}=0.4)$ infection. Figure 2 shows the prevalence of $C$. trachomatis and $N$. gonorrhoeae per anatomic site stratified by age group. The figure shows that the prevalence of anorectal infections is highest in the older age-groups whereas detection of urethral infections remains stable throughout.

The reported number of sexual partners in the period of 6 months prior to consultation was higher by MSM with chlamydia (mean 12 vs. 8.2; $\mathrm{p}=0.03$ ) or gonorrhoea (11 vs. 8.4; $\mathrm{p}=0.03$ ) than those without infection. This was especially related to anorectal infection (13 vs. $8.6 ; p=0.02)$ but not the case for urethral $(p=0.5)$ and 


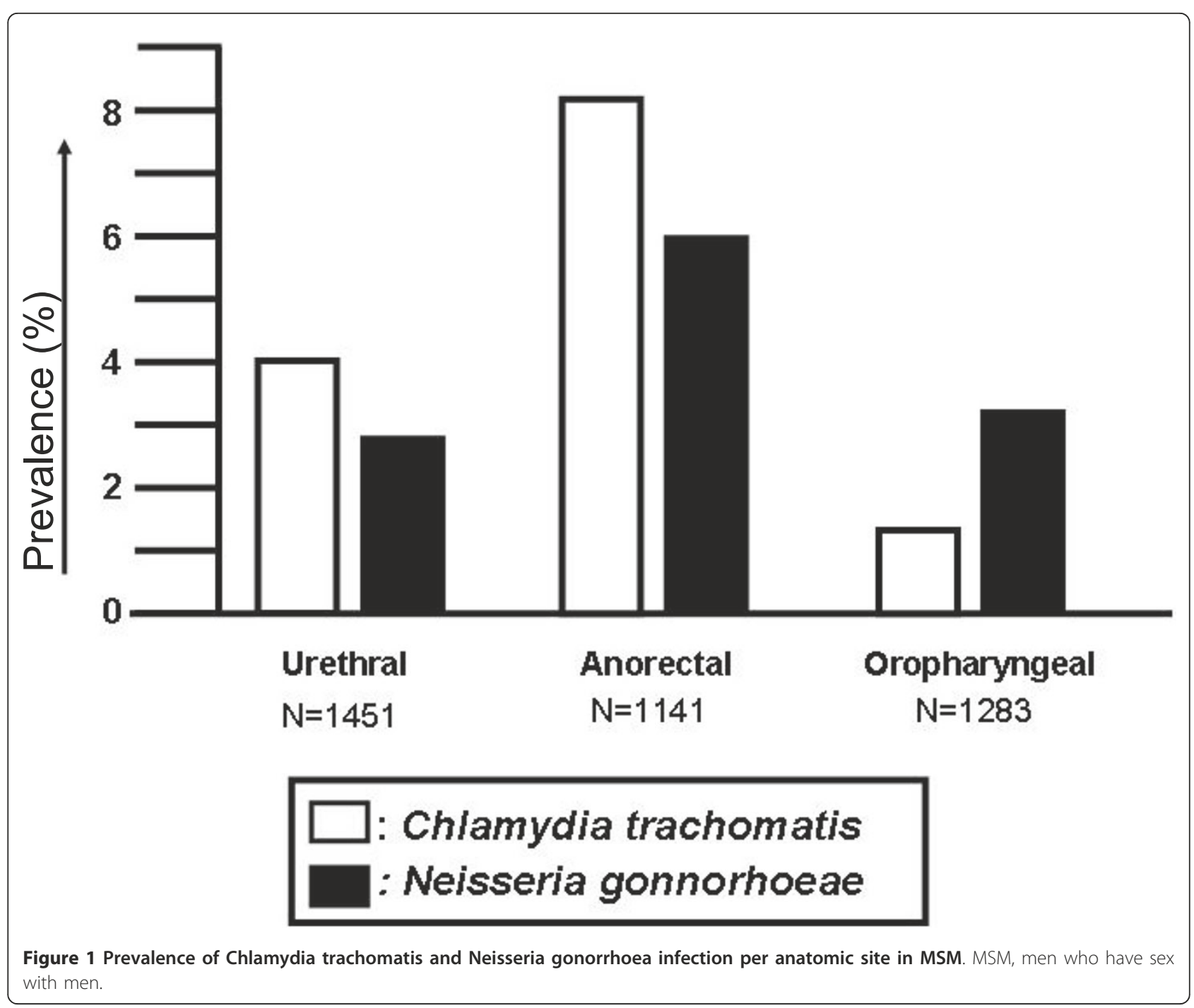

oropharyngeal infection $(\mathrm{p}=0.09)$. In contrast, a history of STI was a risk factor for chlamydia or gonorrhoea infection at each anatomic site: urethra (OR 1.7 (95\% 1.1-2.7); $\mathrm{p}=0.02$ ), oropharynx (OR 2.0 (95\% CI 1.2-3.3); $\mathrm{p}<0.01$ ), and anorectum (OR 2.2 (95\% CI 1.5-2.1); p < 0.001). In those with known HIV status ( $\mathrm{n}=1206)$, HIV infection was associated with increased risk for chlamydia (OR 2.5 (95\% CI 1.6-4.0); p < 0.001) and gonorrhoea (OR 3.6 (95\% CI, 2.2-6.0); p < 0.001). HIV seropositive status was especially associated with anorectal infection (OR 3.6 (95\% CI 2.2-5.6); p < 0.001), to a lesser extent with urethral infection (OR 1.9 (95\% CI 1.0-3.5); $\mathrm{p}=$ $0.04)$ and not with oropharyngeal infection $(\mathrm{p}=0.1)$.

\section{Discussion}

This study shows that testing of multiple anatomic sites for chlamydia and gonorrhoea based on sexual history is a useful strategy for screening for chlamydia and gonorrhoea in MSM. Our data support previous reports and national guidelines that suggest benefit of testing swabs obtained from oropharynx and anorectum in addition to urethral samples in MSM [4-14,21,22].

The prevalence of $C$. trachomatis and $N$. gonorrhoeae infection of oropharynx and anorectum in our study is in line with data reported by others [7-14,21,22]. Similar to those studies, we also found that urethral infections only represent a minority of cases and that anorectal infection is more common. In our setting, the prevalence of chlamydia and gonorrhoea more than doubled with testing of multiple anatomic sites compared to obtained tests for urethral infection alone. The majority of C. trachomatis infections involved a single anatomic site, which was especially the case for anorectal chlamydia, while only a small majority of $N$. gonorrhoea infections involved multiple sites. Altogether, our data strongly support the current guidelines that suggest 


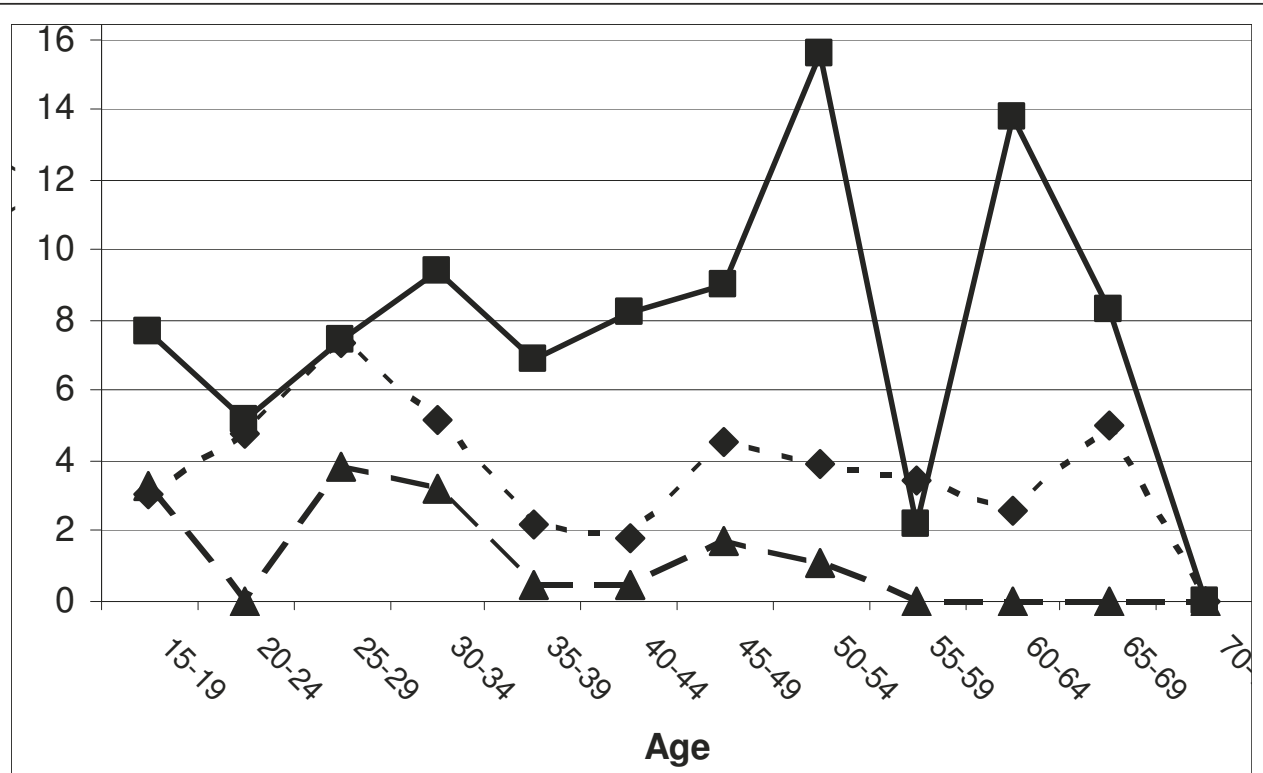

- $\bullet$ - Urethral $\longrightarrow$ Anorectal $\longrightarrow \backsim$ Oropharyngeal

\section{Neisseria gonorroeae infections}

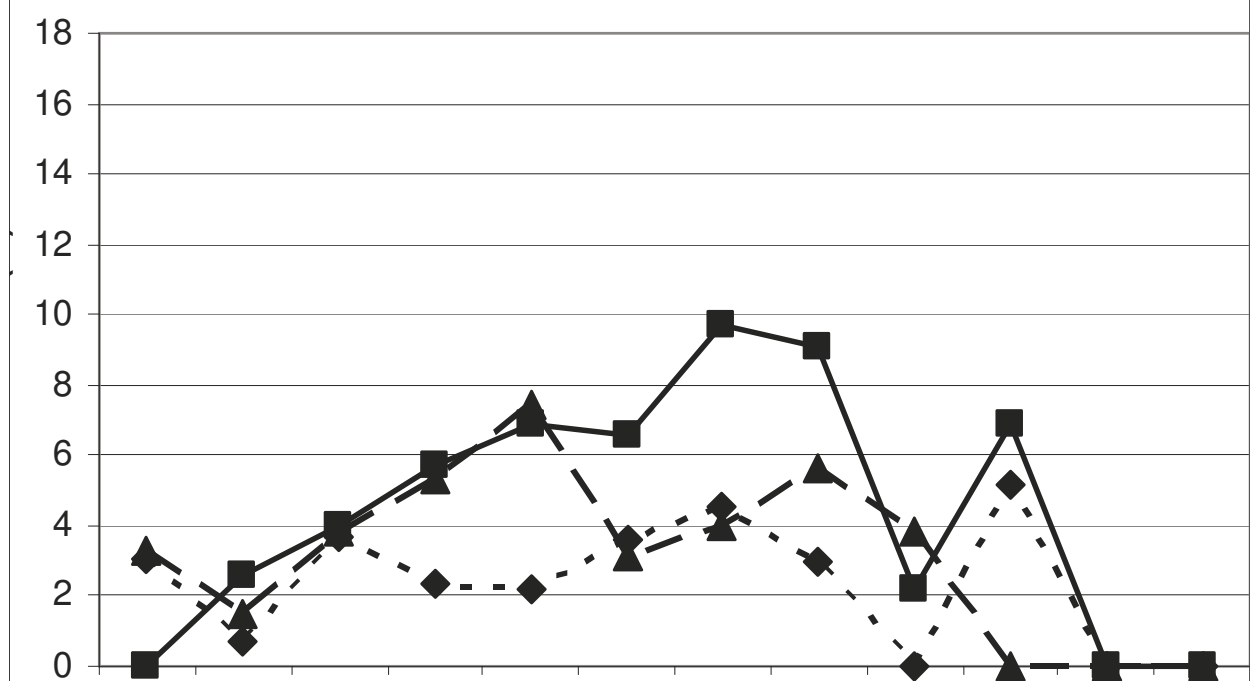

Figure 2 Prevalence of Chlamydia trachomatis and Neisseria gonorrhoeae infection per anatomic site stratified by age groups.

screening anorectum and oropharyngeal samples based on sexual history in addition to urethral tests [4-6,15,16,18].

The prevalence of $C$. trachomatis in oropharyngeal samples was $1.5 \%$ which was in similar range as reported for a cohort of women reporting fellatio in the same setting [24]. Although there is sufficient evidence to screen for oropharyngeal gonorrhoea, there is ongoing debate about the relevance of screening for oropharyngeal Chlamydia [25-28]. This debate is about the prevalence and transmissibility of oropharyngeal C. trachomatis infection. The exact risk of transmission of $C$. trachomatis from throat to penis in fellatio is unknown, but a recent study suggests this may be quite considerable [29]. Altogether, in the absence of clear data about risk of transmission, it seems reasonable to include tests 
for oropharyngeal chlamydia in the routine screening protocol. Oropharyngeal swabs could be tested simultaneously for $N$. gonorrhoeae and $C$. trachomatis if a nuclear amplification test is used. In that regard, costeffectiveness analyses are warranted; a possible method to reduce costs would be to combine swabs from different anatomic sites in a single collection tube and to test these samples simultaneously in a single reaction [30].

The current protocol of opportunistic screening of anatomic sites based on sexual history has a much better performance than theoretically would have been obtained with a screening strategy based on reported symptoms. The vast majority of $C$. trachomatis and $N$. gonorrhoeae infections were asymptomatic, regardless of the anatomic site involved. In addition, the positive predictive values of proctitis and pharyngitis are very low. As such, opportunistic screening is superior to symptom-based screening. We used reported sexual exposure as indication for obtaining anatomic site specific tests. This approach is in line with the current guidelines, but the reliability of sexual history in this context is unclear. Some men may not report exposure for reasons of stigma or embarrassment. An Australian study [31] states that approximately half of the anorectal infections were self-reported, where half were diagnosed by opportunistic screening. Thus an alternative strategy is opportunistic screening of all anatomic sites in all MSM regardless of reported exposure. Considering that the vast majority of our clients reported sexual contact at all three anatomic sites, that MSM can be very outspoken about their sexual practice (e.g. deny ever engaging in receptive anorectal intercourse), that the expected prevalence of infection at anatomic sites that were not exposed during sexual contact is low, and the physical burden of obtaining tests from patients denying sexual contact at that specific body site, we believe that opportunistic screening of multiple anatomic sites in all MSM is unlikely of additional value to sexual-history based screening protocol. Nevertheless, studies are warranted to confirm this hypothesis.

In this retrospective analysis we found that higher number of sexual partners, history of STI, and HIV seropositive status were risk factors for chlamydia and gonorrhoea infection at any anatomic site. These are known risk factors for STI and markers of high risk sexual behaviour. Risk factors associated with anorectal infection were older age, higher number of sexual partners, and HIV seropositive status. Our results show an increasing prevalence of both anorectal chlamydia and gonorrhoea infections with increasing age. As such, specific attention should be given to preventive measures and anorectal screening in MSM with those characteristics.
This study has several limitations. First, one client can be included in the database with multiple visits related to different consultations due to the set-up and anonymous character of the database. Based on some variables (age, postal code, and ethnicity), we estimate that $90 \%$ of the consultations are unique and $10 \%$ are multiple visits. These multiple visits may have introduced some bias when calculating risk factors for chlamydia and gonorrhoea at specific anatomical sites, because those are related to risk behaviour. Secondly, the data presented were collected during routine clinical consultations and only information included in the standard questionnaire was captured systematically. For example, the report of pharyngitis was only recorded if the patient complained about a sore throat, but not specifically asked for. As such, more studies are warranted to confirm our findings.

\section{Conclusions}

Our data indicate that the current strategy of sexual-history based screening of multiple anatomic sites in MSM is a useful and valid guideline for screening for chlamydia and gonorrhoea in MSM and that this approach is superior to a symptom-based screening protocol. Specific attention should be given during consultation to information, prevention and anorectal screening of older MSM, those with a relatively high number of sexual partners, and those with HIV seropositive status.

\section{Acknowledgements}

RPHP presently works for ANOVA Health Institute which is funded by the U. S. President's Emergency Plan for AIDS Relief (PEPFAR) through United States Agency for International Development (USAID) grant number 674-A-00-0800009-00. The aims of the current study are in line of the European EpiGen Chlamydia Consortium of which SAM is member and which is supported by the European Commission within the Sixth Framework Programme through contract number LSHG-CT-2007-037637.

\section{Author details}

${ }^{1}$ The Hague Municipal Health Services, STI clinic, The Hague, The Netherlands. ${ }^{2}$ ANOVA Health Institute, Khutšo Kurhula Offices, Tzaneen, South Africa. ${ }^{3}$ Laboratory of Immunogenetics, Department of Pathology, VU University Medical Center, Amsterdam, The Netherlands. ${ }^{4}$ Department of Medical Microbiology, MCH Westeinde Hospital, The Hague, The Netherlands. ${ }^{5}$ Cluster of Infectious Diseases, Public Health Service Amsterdam, Amsterdam, The Netherlands.

\section{Authors' contributions}

RPHP: study design, data/statistical analyses, drafting the manuscript

SPV: data/statistical analyses, drafting the manuscript

$\mathrm{NN}$ : data collection, critically reading manuscript

SO: data/statistical analyses, drafting the manuscript

JM: data collection, critically reading manuscript

CL: data collection, critically reading manuscript

APL: study design and conception, critically reading manuscript

SAM: study design, conception and coordination, critically revising the manuscript

All authors contributed to writing of the final manuscript.

All authors read and approved the final manuscript 


\section{Competing interests}

The authors declare that they have no competing interests.

Received: 4 March 2011 Accepted: 26 July 2011 Published: 26 July 2011

\section{References}

1. van Valkengoed IG, Morre SA, van den Brule AJ, Meijer CJ, Deville W, Bouter LM, et al: Low diagnostic accuracy of selective screening criteria for asymptomatic Chlamydia trachomatis infections in the general population. Sex Transm Infect 2000, 76(5):375-80

2. Pimenta JM, Catchpole M, Rogers PA, Hopwood J, Randall S, Mallinson H, et al: Opportunistic screening for genital chlamydial infection. Il: prevalence among healthcare attenders, outcome, and evaluation of positive cases. Sex Transm Infect 2003, 79(1):22-7.

3. Honey E, Augood C, Templeton A, Russell I, Paavonen J, Mardh PA, et al: Cost effectiveness of screening for Chlamydia trachomatis: a review of published studies. Sex Transm Infect 2002, 78(6):406-12.

4. Workowski KA, Berman S: Sexually transmitted diseases treatment guidelines, 2010. MMWR Recomm Rep 2010, 17:59(RR-12):1-110.

5. Blystad H, Klouman E: Recommendation for annual HIV and STI testing in MSM introduced in Norway. Euro Surveill 2005, 10(7):E050707.

6. Bourne C, Edwards B, Shaw M, Gowers A, Rodgers C, Ferson M: Sexually transmissible infection testing guidelines for men who have sex with men. Sex Health 2008, 5(2):189-91.

7. Kent CK, Chaw JK, Wong W, Liska S, Gibson S, Hubbard G, et al: Prevalence of rectal, urethral, and pharyngeal chlamydia and gonorrhea detected in 2 clinical settings among men who have sex with men. In Clin Infect Dis. Volume 1:41. San Francisco, California, 2003: 2005:(1):67-74.

8. Annan NT, Sullivan AK, Nori A, Naydenova P, Alexander S, McKenna A, et al: Rectal chlamydia-a reservoir of undiagnosed infection in men who have sex with men. Sex Transm Infect 2009, 85(3):176-9.

9. Gunn RA, O'Brien CJ, Lee MA, Gilchick RA: Gonorrhea screening among men who have sex with men: value of multiple anatomic site testing, San Diego, California, 1997-2003. Sex Transm Dis 2008, 35(10):845-8.

10. Ota KV, Fisman DN, Tamari IE, Smieja M, Ng LK, Jones KE, et al: Incidence and treatment outcomes of pharyngeal Neisseria gonorrhoeae and Chlamydia trachomatis infections in men who have sex with men: a 13year retrospective cohort study. Clin Infect Dis 2009, 1;48(9):1237-43.

11. Papp JR, Ahrens K, Phillips C, Kent CK, Philip S, Klausner JD: The use and performance of oral-throat rinses to detect pharyngeal Neisseria gonorrhoeae and Chlamydia trachomatis infections. Diagn Microbiol Infect Dis 2007, 59(3):259-64.

12. Mimiaga MJ, Helms DJ, Reisner SL, Grasso C, Bertrand T, Mosure DJ, et al: Gonococcal, chlamydia, and syphilis infection positivity among MSM attending a large primary care clinic, Boston, 2003 to 2004. Sex Transm Dis 2009, 36(8):507-11.

13. Schachter J, Moncada J, Liska S, Shayevich C, Klausner JD: Nucleic acid amplification tests in the diagnosis of chlamydial and gonococcal infections of the oropharynx and rectum in men who have sex with men. Sex Transm Dis 2008, 35(7):637-42.

14. Lister NA, Smith A, Tabrizi S, Hayes P, Medland NA, Garland S, et al: Screening for Neisseria gonorrhoeae and Chlamydia trachomatis in men who have sex with men at male-only saunas. Sex Transm Dis 2003, 30(12):886-9.

15. Carder C, Mercey D, Benn P: Chlamydia trachomatis. Sex Transm Infect 2006, 82(Suppl 4):iv10-iv12.

16. Bignell C, Ison CA, Jungmann E: Gonorrhoea. Sex Transm Infect 2006, 82(Suppl 4):iv6-iv9.

17. Ross JD, Ison CA: Introduction and summary tables. Sex Transm Infect 2006, 82(Suppl 4):iv1-iv5.

18. Nederlandse Vereniging van Dermatologie \& Venereologie: Diagnostiek en behandeling van seksueel overdraagbare aandoeningen (SOA)-korte samenvatting SOA richtlijn., 1-8-2008.

19. Spaargaren J Schachter J, Moncada J, de Vries HJ, Fennema HS, Pena AS, et al: Slow epidemic of lymphogranuloma venereum L2b strain. Emerg Infect Dis 2005, 11(11):1787-8.

20. Centers for Disease Control and Prevention (CDC): Clinic-based testing for rectal and pharyngeal Neisseria gonorrhoeae and Chlamydia trachomatis infections by community-based organizations -five cities, United States, 2007. MMWR Morb Mortal Wkly Rep 2009, 58:716-9.
21. Benn PD, Rooney G, Carder C, Brown M, Stevenson SR, Copas A, et al: Chlamydia trachomatis and Neisseria gonorrhoeae infection and the sexual behaviour of men who have sex with men. Sex Transm Infect 2007, 83(2):106-12.

22. Ostergaard L, Agner T, Krarup E, Johansen UB, Weismann K, Gutschik E: PCR for detection of Chlamydia trachomatis in endocervical, urethral, rectal, and pharyngeal swab samples obtained from patients attending an STD clinic. Genitourin Med 1997, 73(6):493-7.

23. Morre SA, Spaargaren J, Fennema JS, de Vries HJ: Molecular diagnosis of lymphogranuloma venereum: PCR-based restriction fragment length polymorphism and real-time PCR. J Clin Microbiol 2005, 43(10):5412-3.

24. Peters RPH, Nijsten N, Mutsaers J, Jansen CL, Morré SA, van Leeuwen AP: Screening of oropharynx and anorectum increases prevalence of Chlamydia trachomatis and Neisseria gonorrhoeae infection in female STD clinic visitors (ACCEPTED FOR PUBLICATION). Sex Transm Dis 2010

25. Stamm W: Chlamydia trachomatis infections in the adult. In Sexually Transmitted Diseases.. 4 edition. Edited by: Holmes KSPSWeae. New York: McGraw-Hill: 2008 .

26. Hook E3HH: Gonococcal infections in the adult. In Sexually Transmitted Diseases.. 4 edition. Edited by: Holmes KSPSWeae. New York: McGraw-Hill; 2008:

27. Lafferty WE, Hughes JP, Handsfield HH: Sexually transmitted diseases in men who have sex with men. Acquisition of gonorrhea and nongonococcal urethritis by fellatio and implications for STD/HIV prevention. Sex Transm Dis 1997, 24(5):272-8.

28. Janier M, Lassau F, Casin I, Morel P: Pharyngeal gonorrhoea: the forgotten reservoir. Sex Transm Infect 2003, 79(4):345.

29. Bernstein KT, Stephens SC, Barry PM, Kohn R, Philip SS, Liska S, et al: Chlamydia trachomatis and Neisseria gonorrhoeae transmission from the oropharynx to the urethra among men who have sex with men. Clin Infect Dis 2009, 15;49(12):1793-7.

30. Morre SA, van DR, Meijer CJ, van den Brule AJ, Kjaer SK, Munk C: Pooling cervical swabs for detection of Chlamydia trachomatis by PCR: sensitivity, dilution, inhibition, and cost-saving aspects. J Clin Microbiol 2001, 39(6):2375-6.

31. Jin F, Prestage GP, Mao L, Kippax SC, Pell CM, Donovan B, et al: Incidence and risk factors for urethral and anal gonorrhoea and chlamydia in a cohort of HIV-negative homosexual men: the Health in Men Study. Sex Transm Infect 2007, 83(2):113-9.

\section{Pre-publication history}

The pre-publication history for this paper can be accessed here: http://www.biomedcentral.com/1471-2334/11/203/prepub

\section{doi:10.1186/1471-2334-11-203}

Cite this article as: Peters et al:: Evaluation of sexual history-based screening of anatomic sites for chlamydia trachomatis and neisseria gonorrhoeae infection in men having sex with men in routine practice. BMC Infectious Diseases 2011 11:203.

\section{Submit your next manuscript to BioMed Central and take full advantage of:}

- Convenient online submission

- Thorough peer review

- No space constraints or color figure charges

- Immediate publication on acceptance

- Inclusion in PubMed, CAS, Scopus and Google Scholar

- Research which is freely available for redistribution

Submit your manuscript at www.biomedcentral.com/submit
C Biomed Central 